\title{
Modified axial computed tomography classification of cervical ossification of the posterior longitudinal ligament: selecting the optimal operating procedure and enhancing the accuracy of prognosis
}

\author{
Tuo Shao ${ }^{\#}$, Jiao Gu\#, Yigeng Zhu, Weilong Tang, Qingsong Li, Juncheng Lu, Yuhang Hu, Zhange Yu, \\ Hongtao Shen
}

Department of Spinal Surgery, The First Affiliated Hospital of Harbin Medical University, Harbin, China

\#These authors contributed equally to this work.

Correspondence to: Hongtao Shen, Zhange Yu. Department of Spinal Surgery, The First Affiliated Hospital of Harbin Medical University, no. 23 Youzheng Street, Harbin 150001, China. Email: 982575101@qq.com; zhangeyu1967@aliyun.com.

Background: Cervical ossification of the posterior longitudinal ligament (OPLL) causes spinal cord compression, which can lead to myelopathy or radiculopathy. Non-surgical treatments have little effect on this condition. Current OPLL classification systems offer little guidance on the selection of an appropriate operating procedure. In this study, we developed a modified axial computed tomography classification (MACTC) scheme. We then examined the usefulness of the MACTC scheme and two other existing classification schemes in guiding OPLL operation choice.

Methods: Following screening in which a defined exclusion criteria was used, a total of 91 patients with OPLL participated in the study. Patients' follow-up data for at least 2 years were obtained. The recovery rate of the Japanese Orthopaedic Association (JOA) scores was compared to two other classification schemes.

Results: According to the MACTC, central-sharp-type OPLL had a lower recovery rate of the JOA score than that of central-gentle-type OPLL $(36.05 \pm 32.38$ vs. $83.90 \pm 23.52, \mathrm{P} \leq 0.05)$. The recovery rate of the JOA scores in the ipsilateral open-door OPLL group was significantly lower than that in the contralateral group of the lateral-steep type $(36.67 \pm 41.5$ vs. $88.89 \pm 17.21, \mathrm{P}=0.04)$, but not of that in the lateral-gentle type. There was no significant difference in the recovery rates of the JOA scores between groups when using either existing classification scheme $(\mathrm{P}>0.05)$.

Conclusions: The MACTC scheme can assist surgeons to choose the most appropriate operating procedure, and provide an accurate prognosis. If operations on central-sharp-type OPLL are not performed using both the posterior and anterior approaches, prognosis will be poor. The contralateral side should be the first choice for door opening in laminoplasty, especially for patients with lateral-steep-type OPLL. Severe OPLL may not be an absolute contraindication for the posterior approach.

Keywords: Ossification of the posterior longitudinal ligament (OPLL); classification; posterior approach; laminoplasty

Submitted Jul 13, 2020. Accepted for publication Nov 20, 2020.

doi: 10.21037 /qims-20-862

View this article at: http://dx.doi.org/10.21037/qims-20-862

^ ORCID: 0000-0003-3774-4778. 


\section{Introduction}

Ossification of the posterior longitudinal ligament (OPLL) is a cervical degenerative disease that causes spinal cord compression. OPLL is characterized by ectopic bone formation in the spinal ligament, which can result in myelopathy or radiculopathy. Non-surgical treatments have little effect on this condition (1). Various surgical procedures exist for treating cervical OPLL, including the anterior approach, posterior approach, and an approach that combines both the anterior and posterior approaches (2). The anterior approach is suitable for single-segment OPLL; however, the incidence of complications is high (3). The routine protocol for multi-segment continuous OPLL uses the posterior approach, which includes laminoplasty, laminectomy, and laminectomy with fusion. As one of the commonly used surgical procedures, laminoplasty has less complications; however, its effects can be limited by the reduced space for indirect decompression (4). The combined anterior-posterior approach is suitable for patients with continuous ossification for which the degree of ossification is more than $60 \%$ (5). In addition to the degree of ossification, the axial morphology of ossification also affects the severity of symptoms and the resulting prognosis for OPLL (6).

Numerous classification systems for OPLL exist in academic circles. Most of these systems describe and classify the shape of the ossification, but provide little guidance on the appropriate selection of an operating procedure. The value of classification schemes lies primarily in the provision of treatment guidance and the accurate prediction of prognosis. By examining the long-term follow-up data of patients with OPLL and undertaking a retrospective analysis of their clinical data, we were able to further refine and improve the axial CT classification of OPLL that was proposed by the Investigation Committee on OPLL of the Japanese Ministry of Public Health and Welfare (7). The modified axial computed tomography classification (MACTC) provides a comprehensive and detailed description of the morphological characteristics of OPLL. This paper also examines the guiding role of MACTC in OPLL therapy and the prediction of prognosis.

\section{Methods}

\section{Inclusion and exclusion criteria}

To be eligible to participate in this study, patients needed to meet the following inclusion criteria: (I) diagnosed with OPLL, as confirmed by computed tomography (CT);
(II) meeting the criteria for use of the posterior approach (i.e., range of ossification $\geq$ three segments, continuous or not); (III) symptoms of myelopathy [i.e., the magnetic resonance imaging (MRI) scans indicating cervical spinal cord compression, primarily due to OPLL]; (IV) treated in our department with a unilateral open-door laminoplasty between May 2014 and May 2018; (V) completed continuous follow-up for more than 2 years. Patients were excluded from the study if they met any of the following exclusion criteria: (I) a myelopathy not attributable to OPLL; (II) a medical history of cervical trauma, ankylosing spondylitis, infection, or deformity; (III) inability to tolerate surgery; (IV) history of revision operations; and/or (V) presence of cervical kyphosis (i.e., K-line negative).

\section{Modified axial CT classification}

The primary classification of central-type, lateral-type, or flat-type OPLL was based on the distribution of ossification. Patients with central-type OPLL had a protuberant apex on the dorsal side of the ossification, located in the middle third of the spine canal; those with lateral type OPLL had a protuberance located in either of the two outer thirds of the spine canal; those with flat-type OPLL had an ossification without a protuberance. In relation to central-type OPLL, a secondary classification was made based on the sharpness of the ossification at the highest point of the bulge, such that patients were classified as having either central-sharp-type OPLL or central-gentle-type OPLL. In relation to lateral-type OPLL, a secondary classification was made based on the difference in the proportion of bilateral ossification in the median diameter of the vertebral canal on the CT axial position, such that patients were classified as having either lateral-steep-type OPLL (with the difference between the mild and severe side $\geq 30 \%$ ) or lateral-gentle-type OPLL (with the difference between the mild and severe side $<30 \%$ ) (Figure 1).

\section{Existing classifications of OPLL}

Under established classifications of axial ossification morphology, cases can be classified into three types: (I) the hill type, which has a wide base and a narrow free end; (II) the mushroom type, which has a narrow base and a wide free end; and (III) the square type, which has a wide base and a wide free end (8) (Figure 2A). In the present study, cases were also classified as boomerang, teardrop, or triangular, according to the structure of the compressed spinal cord as recorded on $\mathrm{T} 2$-weighted axial images (Figure 2B), with the concomitant spinal cord compression 

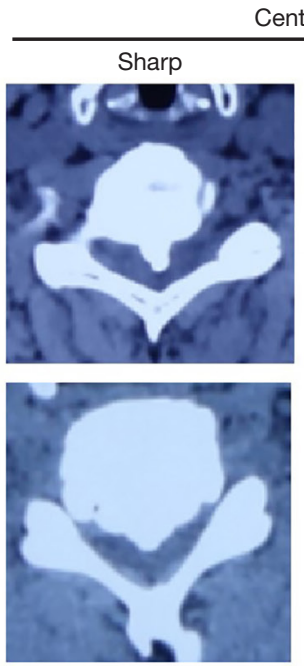

Central Type
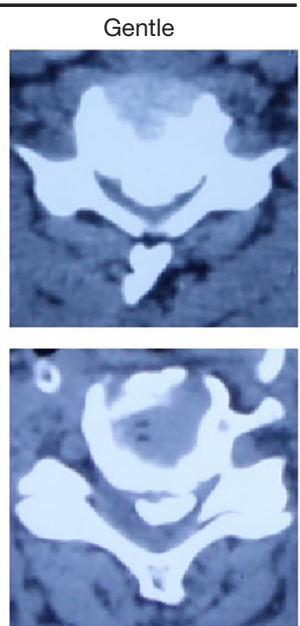

Flat Type
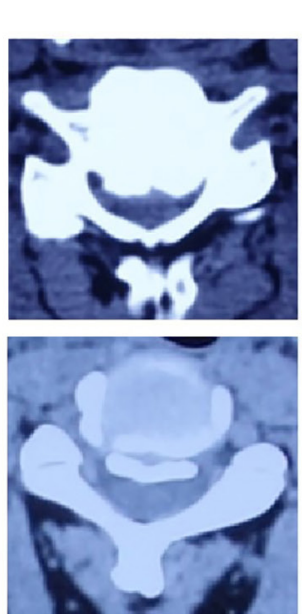
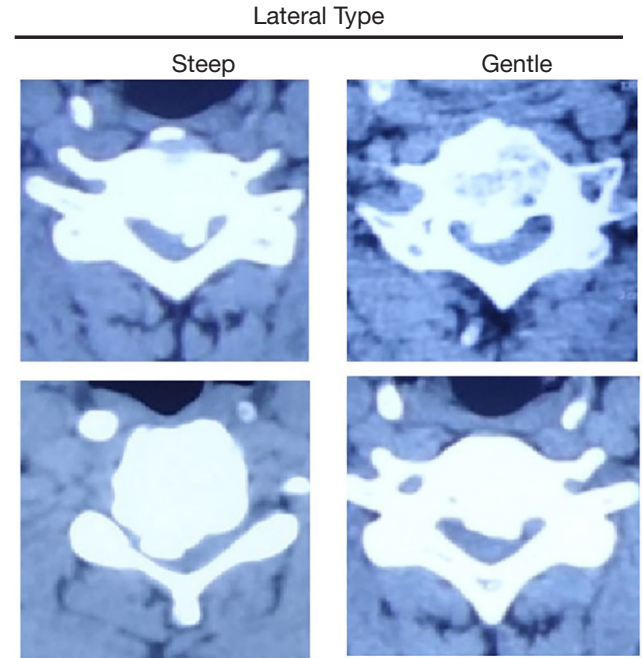

Figure 1 Examples of the modified axial computed tomography classification.

A

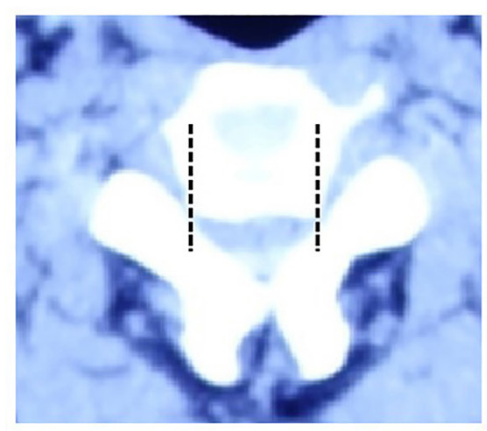

B

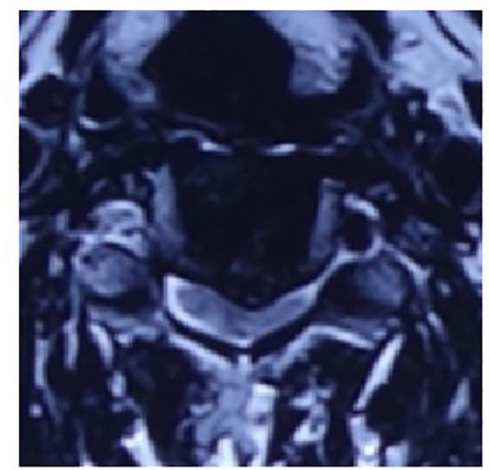

Mushroom

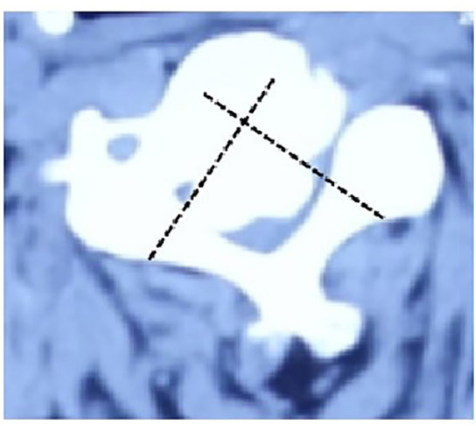

Teardrop

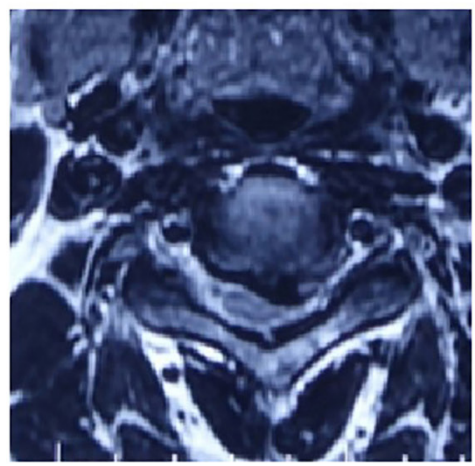

Hill

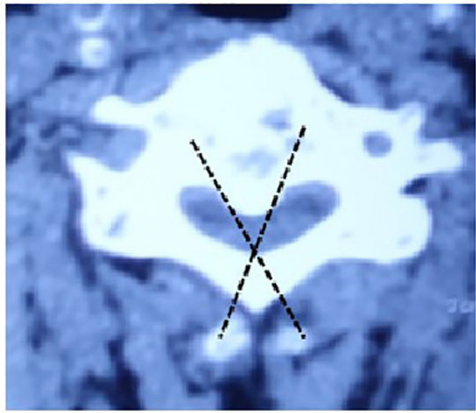

Triangular

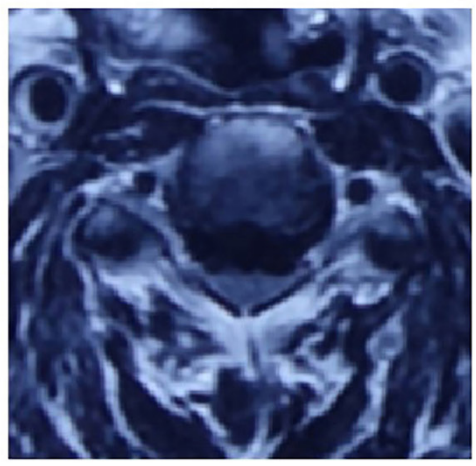

Figure 2 Examples of established classifications. (A) Three types of axial ossification morphological classification. (B) Three types of axial MRI-T2 spinal cord classification. 

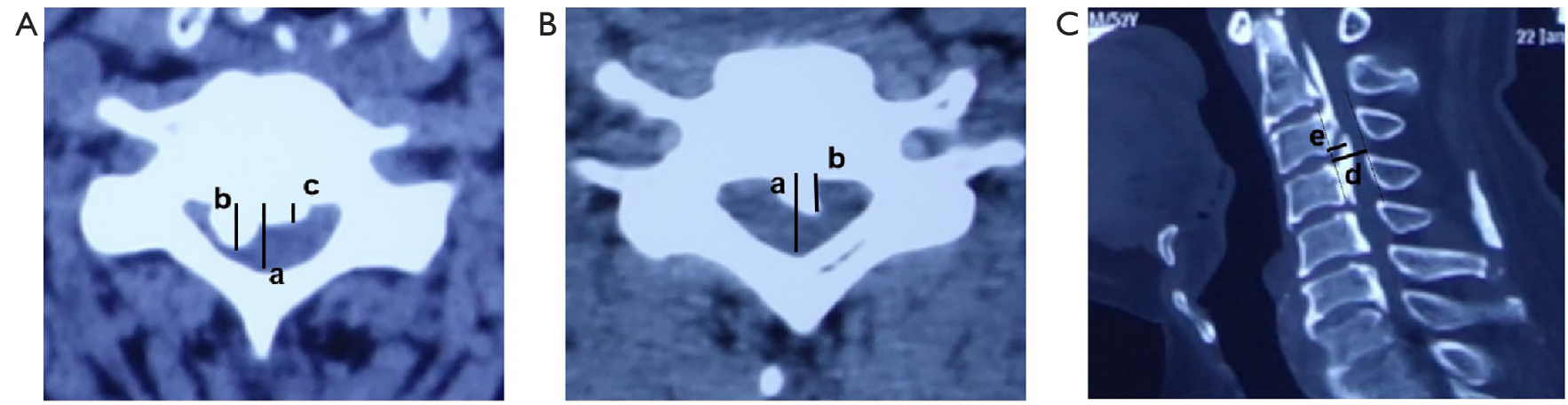

Figure 3 Diagram of measurement methods. (A,B) The ratio of ossification to axial spinal canal diameter (ROASCD) was calculated using the following formula: ROASCD $=\mathrm{b} / \mathrm{a}^{*} 100 \%$. The difference of ROASCD between the mild and severe side was calculated using the following equation: (b-c)/a*100\%. (C) The ROSSCD was calculated using the following equation: ROSSCD $=e / \mathrm{d}^{*} 100 \%$.

increasing sequentially (9).

\section{Surgical techniques}

Surgery was performed according to the modified Hirabayashi method (10). In accordance with this method, each patient was placed in the prone position, a middle incision using the posterior cervical approach was made, the paravertebral muscles were detached, and the spinous process was removed. The border of the lamina and the lateral mass was ground thin using a speed burr. A rongeur was used to excise a unilateral groove, forming the hinge side. The other side was penetrated completely to form the door opening side, which was fixed using a micro titanium plate and screws.

\section{Clinical data}

The Clinical Research Ethics Committee approved the protocol. The clinical data of the patients were analyzed retrospectively. The basic information collected included age, gender, duration, follow-up time, operation time, and blood loss. Symptomatic improvement was evaluated using the following metrics: the pre-operative Japanese Orthopaedic Association (JOA) score, the postoperative JOA score, the JOA recovery rate (JOARR), and the half-year and 1-year relief (HYR, OYR) ratings. Imaging data, including data of the spinal cord area (SCA), the ratio of ossification to sagittal spinal canal diameter (ROSSCD), and the axial spinal canal diameter (ROASCD, were measured using Camera Measure software (v2.1.3.250, e2eSoft; Figure 3). The JOARR was calculated using the following formula: recovery rate $(\%)=$ (postoperative $\mathrm{JOA}-$ pre-operative
$\mathrm{JOA}) /(17$ full score - pre-operative JOA $) \times 100 \%$. HYR and OYR were defined as the relief in symptoms patients reported experiencing at the half- and 1-year follow-up time points; patients who reported a significant improvement (JOARR $\geq 50 \%$ ) were defined as positive; otherwise, they were defined as negative.

A total of 132 patients with OPLL were assessed. After a screening process in which the exclusion criteria (see above) were applied, 91 patients remained. Three classification schemes were then used to classify OPLL (i.e., the MACTC and two other established classification systems). Two evaluators with similar training classified each case independently. If opinions differed, a third senior evaluator made the final decision. The two observers were tested again after 1 week. The intra- and interobserver consistency of MACTC was analyzed using a Cohen's kappa test. To fully evaluate any postoperative effects, cases of lateraltype OPLL were partitioned into either the ipsilateral group or the contralateral group based on the nature of the open door and ossification sides. Patients with a ROASCD $\geq 60 \%$ were placed into the severe group, and those with a ROASCD $<60 \%$ were placed into the mild group. The HYR and OYR rates of the groups were also evaluated.

\section{Statistical analysis}

SPSS 23.0 statistical software (IBM, Armonk, NY, USA) was used to conduct the statistical analysis. Graphpad Prism 7.0 (GraphPad Software, Inc., San Diego, CA, USA) was used to prepare the graphs. Measurement data are expressed as mean \pm standard deviation (SD). Depending on the sample size, a D'Agostino and Pearson normality 
Table 1 Clinical data of cases according to modified axial computed tomography classification

\begin{tabular}{|c|c|c|c|c|c|c|c|}
\hline Variable & $\begin{array}{c}\text { Flat-type mean } \\
(\mathrm{SD})(\mathrm{n}=22)\end{array}$ & \multicolumn{3}{|c|}{ Central-type, mean (SD) } & \multicolumn{3}{|c|}{ Lateral-type, mean (SD) } \\
\hline Age (year) & $57.5( \pm 8.227)$ & $52.23( \pm 7.96)$ & $57.54( \pm 8.55)$ & $56.69( \pm 7.91)$ & $53.36( \pm 4.13)$ & $56.85( \pm 7.92)$ & $55.25( \pm 6.58)$ \\
\hline Female (\%) & 18.18 & 40.00 & 28.57 & 31.11 & 27.27 & 23.08 & 25.00 \\
\hline Duration (month) & $9.78( \pm 14.18)$ & $4.95( \pm 5.14)$ & $18.80( \pm 31.37)$ & $15.72( \pm 31.37)$ & $4.20( \pm 7.02)$ & $12.79( \pm 23.38)$ & $8.85( \pm 18.05)$ \\
\hline Post-JOA (/17) & $15.86( \pm 2.05)$ & $14.70^{\mathrm{a}}( \pm 1.89)$ & $16.09^{\mathrm{a}}( \pm 1.69)$ & $15.78( \pm 1.81)$ & $16.09( \pm 0.94)$ & $16.23( \pm 1.01)$ & $16.17( \pm 0.96)$ \\
\hline JOA-R (\%) & $74.36( \pm 35.95)$ & $36.05^{\mathrm{a}}( \pm 32.38)$ & $83.90^{\mathrm{a}}( \pm 23.52)$ & $73.27( \pm 32.35)$ & $65.15( \pm 39.76)$ & $79.92( \pm 30.73)$ & $73.15( \pm 35.16)$ \\
\hline Recovery time (month) & $10.00( \pm 7.22)$ & $11.78( \pm 7.79)$ & $10.63( \pm 11.03)$ & $10.86( \pm 10.38)$ & $9.73( \pm 10.97)$ & $6.92( \pm 6.39)$ & $8.21( \pm 8.70)$ \\
\hline Follow-up time (year) & $2.91( \pm 1.57)$ & $3.62( \pm 1.67)$ & $2.90( \pm 1.56)$ & $3.06( \pm 1.59)$ & $3.03( \pm 1.50)$ & $3.36( \pm 1.97)$ & $3.21( \pm 1.74)$ \\
\hline $\mathrm{SAC}(\mathrm{mm})$ & $1.72( \pm 1.15)$ & $2.10( \pm 1.37)$ & $2.12( \pm 1.50)$ & $2.11( \pm 1.45)$ & $1.85( \pm 1.38)$ & $1.49( \pm 1.21)$ & $1.65( \pm 1.27)$ \\
\hline ROSSCD (\%) & $48.89( \pm 9.47)$ & $48.92( \pm 12.86)$ & $50.97( \pm 13.44)$ & $50.52( \pm 13.19)$ & $52.64( \pm 12.07)$ & $48.66( \pm 13.51)$ & $50.48( \pm 12.75)$ \\
\hline ROASCD (\%) & $\begin{array}{l}49.74^{\text {b.c }} \\
( \pm 10.26)\end{array}$ & $\begin{array}{c}58.71 \\
( \pm 11.89)\end{array}$ & $\begin{array}{c}57.45 \\
( \pm 14.31)\end{array}$ & $\begin{array}{c}57.73^{\mathrm{b}} \\
( \pm 13.69)\end{array}$ & $\begin{array}{c}59.46 \\
( \pm 10.09)\end{array}$ & $\begin{array}{c}55.12 \\
( \pm 17.04)\end{array}$ & $\begin{array}{c}57.11^{\mathrm{c}} \\
( \pm 14.16)\end{array}$ \\
\hline C2-7 Cobb angle ( $\left.{ }^{\circ}\right)$ & $16.64( \pm 7.71)$ & $19.30( \pm 15.81)$ & $15.04( \pm 9.41)$ & $16.31( \pm 11.18)$ & $12.97( \pm 7.44)$ & $16.72( \pm 9.13)$ & $15.00( \pm 8.44)$ \\
\hline
\end{tabular}

${ }^{a}$, statistical significance between the central-sharp and central-gentle groups $(P \leq 0.01)$; ${ }^{b}$, statistical significance between the flat and central groups $(\mathrm{P} \leq 0.01)$; ${ }^{c}$, statistical significance between the flat and lateral groups $(\mathrm{P} \leq 0.01)$. SD, standard deviation; Pre-JOA, preoperative Japanese Orthopaedic Association scores; post-JOA, postoperative JOA scores; SAC, space available for the cord; ROSSCD, ratio of ossification to sagittal spinal canal diameter; ROASCD, ratio of ossification to axial spinal canal diameter.

test or a Kolmogorov-Smirnov (KS) normality test was used to assess the homogeneity of the normal distribution and the variance. Normal distributions were tested using a $t$-test or a corrected $t$-test between groups, according to the homogeneity of the variance. Non-normal distributions were tested using a Mann-Whitney $\mathrm{U}$ test. Chi-squared tests were conducted to examine the categorical variables. A rank-sum test was used for the ranked data. For non-linear fitting, a second-order polynomial (quadratic) method was employed. A probability value of $\mathrm{P}<0.05$ was considered statistically significant.

\section{Results}

\section{Central-sharp-type OPLL had poor prognosis}

As stated above, patients were classified into one of three groups using the MACTC scheme. Of these patients, 22 patients had flat-type OPLL, 45 had central-type
OPLL (10 of whom had central-sharp-type OPLL and 35 of whom had central-gentle-type OPLL), and 24 had lateral-type OPLL (11 of whom had lateral-steep-type OPLL and 13 of whom had lateral-gentle-type OPLL). There was no significant difference $(\mathrm{P}>0.05)$ in age, gender, duration, follow-up time, operation time, blood loss, SAC, ROSSCD, or the C2-7 Cobb angle between groups at the same level (Table 1). The ROASCD for flattype OPLL $(49.74 \pm 10.26)$ was significantly higher than that of central-type OPLL $(57.73 \pm 13.69, \mathrm{P}=0.018)$ and lateral-type OPLL (57.11 $\pm 14.16, \mathrm{P}=0.033)$. No significant difference was found in post-JOA scores and JOARR between the three groups in the primary classification, including between the lateral-gentle and lateral-steep categories at the secondary level of classification. However, the post-JOA score and JOARR of central-sharp-type OPLL $(14.7 \pm 1.889, \mathrm{P}<0.01 ; 36.05 \pm 32.38, \mathrm{P}<0.001)$ were significantly lower than those of the central-gentle-type OPLL $(16.09 \pm 1.687,83.9 \pm 23.52)$. There was strong 
interobserver $(\mathrm{kappa}=0.896, \mathrm{P}<0.001)$ and intra-observer (kappa $1=0.970, \mathrm{P}<0.001 ;$ kappa $2=0.985, \mathrm{P}<0.001)$ consistency in the MACTC scheme (see Table 2).

\section{Post-operative effect of the open-side type and severity of ossification}

The JOARR of the ipsilateral group $(36.67 \pm 41.5)$ was significantly lower than that of the contralateral group $(88.89 \pm 17.21, \mathrm{P}=0.043)$ with lateral-steep-type OPLL; however, different results were found for patients with lateral-gentle-type OPLL (see Table 3). The HYR-positive rate in the severe group was significantly lower than that in the mild group (Chi-square $=5.095, \mathrm{P}=0.024)$, but there was no significant difference in the OYR rates (Table 4).

\section{Assessment of established classification systems}

According to axial ossification morphology classification, 59 cases were classified as hill, 2 cases as mushroom, and 30 cases as square. The spinal cord compression classification categorized 40 cases as boomerang, 29 patients as teardrop, and 22 as triangular. There was no statistically significant difference in the JOARR values between the groups in the two evaluated classification systems $(\mathrm{P}>0.05$; Table 5$)$.

\section{Discussion}

When evaluating the relationship between OPLL morphology and prognosis, it is generally thought that lateral-type OPLL is more likely to lead to neurological

Table 2 Consistency tests for observers

\begin{tabular}{llll}
\hline Consistency & Kappa value & Standard error & $P$ value \\
\hline Intra-observer 1 & 0.970 & 0.021 & $<0.001$ \\
Intra-observer 2 & 0.985 & 0.015 & $<0.001$ \\
Interobserver & 0.896 & 0.037 & $<0.001$ \\
\hline
\end{tabular}

symptoms than central-type OPLL (11). The mechanical analysis showed that compared to central-type OPLL, flattype OPLL caused the greatest compression of the spinal cord, while lateral-type OPLL caused the greatest increase in traction of the nerve root (12). Patients with centralgentle-type OPLL showed more improved outcomes than those with central-sharp-type OPLL. Despite no statistically significant differences existing between the groups in the primary classification of MACTC, after further refinement of the classification, the retrospective analysis showed that the post-JOA score and JOARR for central-sharp-type OPLL were significantly lower than those for central-gentle type OPLL after unilateral opendoor laminoplasty. It has been suggested that sagittal beak type ossification is a poor prognostic indicator of thoracic OPLL outcomes $(13,14)$, and that this indirectly proves that ossifications with sharp profiles clearly cause harm post-operatively. Laminoplasty may have a poor effect on central-sharp-type ossifications due to the limited contact area between the sharp ossification and the dura (i.e., the expansion of the dura and spinal cord is poor because the contact area does not provide a sufficient reaction force for the dura). However, to date, no evidence has been found to confirm this hypothesis; thus, further mechanical research needs to be undertaken. In relation to the poor prognosis, central-sharp-type OPLL has been classified as a distinct type, as it cannot be resolved by posterior cervical laminoplasty. A combination of posterior and anterior decompression surgery is necessary to completely remove

Table 4 Postoperative relief of severe and mild ossification

\begin{tabular}{lccccc}
\hline \multirow{2}{*}{ ROASCD } & \multicolumn{2}{c}{ Half-year relief * } & & \multicolumn{2}{c}{ One-year relief } \\
\cline { 2 - 3 } \cline { 5 - 6 } & Positive & Negative & & Positive & Negative \\
\hline Severe $(\geq 60 \%)$ & 14 & 26 & & 31 & 9 \\
Mild $(<60 \%)$ & 30 & 21 & & 42 & 9 \\
\hline * statistical significance (P $\leq 0.05)$. & ROASCD, ratio of ossification \\
to axial spinal canal diameter.
\end{tabular}

Table 3 Postoperative effects of different open sides

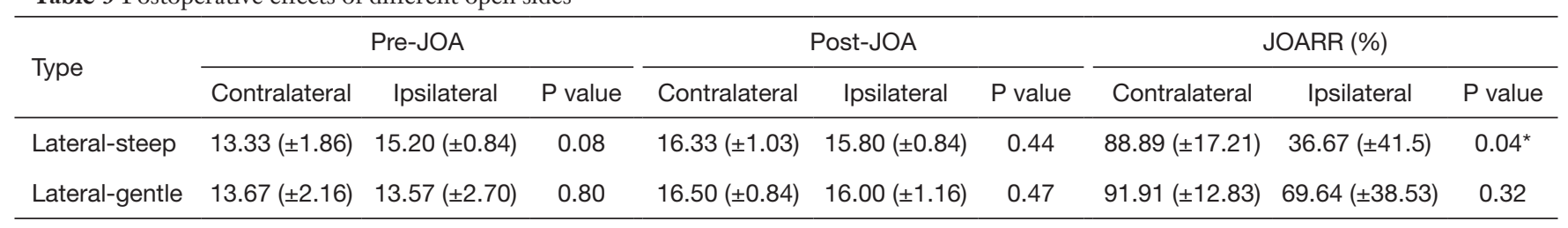

*, statistical significance $(\mathrm{P} \leq 0.05)$. JOARR, Japanese Orthopaedic Association recovery rate. 
Table 5 Postoperative effect according to classic classifications

\begin{tabular}{lccccccc}
\hline & \multicolumn{2}{c}{ Axial CT ossification morphology classification } & & \multicolumn{3}{c}{ MRI spinal cord compression classification } \\
\cline { 2 - 3 } & Hill $(n=59)$ & Square $(n=30)$ & Mushroom $(n=2)$ & & Teardrop $(n=29)$ & Triangular $(n=22)$ & Boomerang $(n=40)$ \\
\hline Pre-JOA & $13.31( \pm 3.17)$ & $12.80( \pm 3.84)$ & $11.50( \pm 4.95)$ & & $13.72( \pm 2.20)$ & $12.32( \pm 4.38)$ & $13.08( \pm 3.51)$ \\
Post-JOA & $16.02( \pm 1.28)$ & $15.73( \pm 2.32)$ & $15.00( \pm 1.41)$ & & $16.21( \pm 0.94)$ & $15.18( \pm 2.61)$ & $16.08( \pm 1.37)$ \\
JOARR $(\%)$ & $73.92( \pm 32.63)$ & $73.69( \pm 36.80)$ & $58.34( \pm 11.79)$ & & $73.07( \pm 34.44)$ & $66.82( \pm 36.21)$ & $77.49( \pm 31.74)$ \\
\hline
\end{tabular}

CT, computed tomography; MRI, magnetic resonance imaging; Pre-JOA, pre-operative Japanese Orthopaedic association scores; PostJOA, postoperative JOA scores; JOARR, JOA score recovery rate.
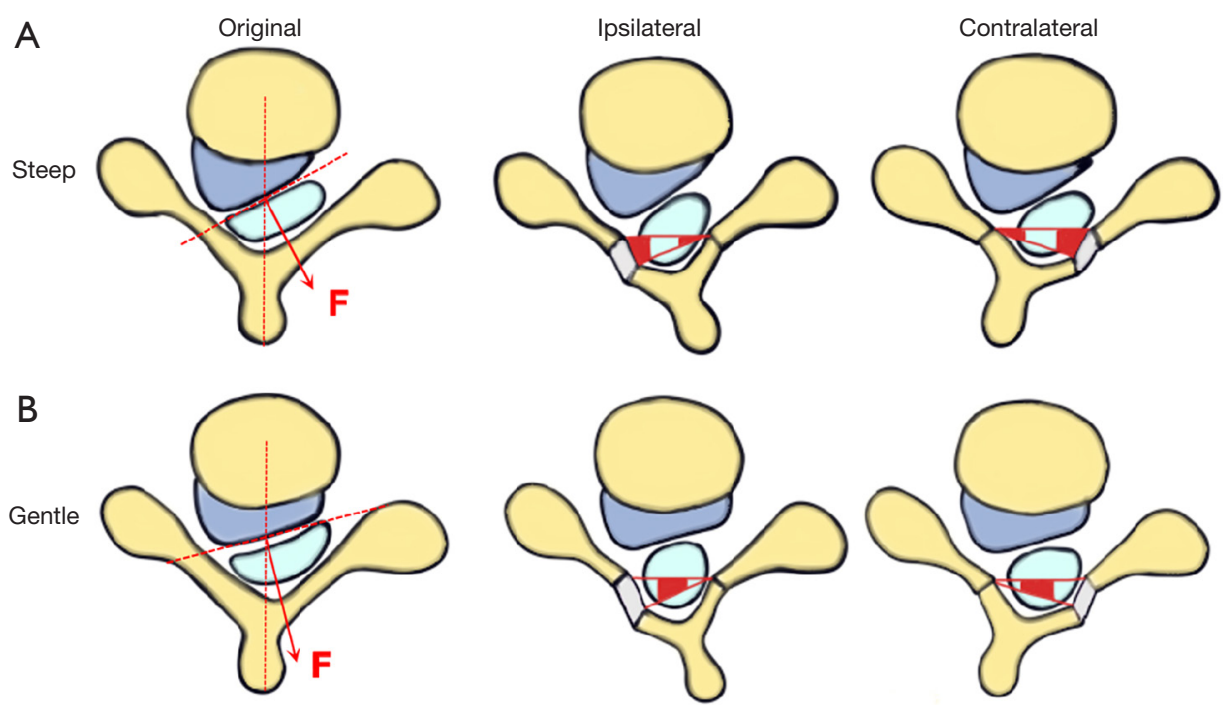

Figure 4 Diagram showing increased space for lateral type OPLL on different open sides after unilateral open-door laminoplasty. (A) Lateral-steep-type OPLL. (B) Lateral-gentle-type OPLL. OPLL, ossification of the posterior longitudinal ligament.

the pressure-inducing sharp ossification.

It has been reported that the post-operative effects of cervical unilateral open-door laminoplasty may be related to changes in the space available for the cord (SAC) (15). Except for the opening angle and the degree of ossification, the expansion of the spinal cord after laminoplasty depends on the contact area between the ossification and the stress surface of the dura. The available area of the dura was sufficient for flat-type OPLL, central-gentle-type OPLL, and lateral-type OPLL (i.e., it allowed the rebound reaction force to dissipate properly and thus for the spinal cord to expand immediately or subsequently). Conversely, the duraossification contact area of central-sharp-type OPLL was small, which prevented the compression force from acting evenly on the dura after opening the door, which resulted in low spinal cord expansion. Additionally, the large resulting pressure might have caused secondary damage during dural expansion.

The choice of side in an open-door procedure has remained controversial for lateral ossification. We compared the postoperative effect of different open-door sides in terms of the secondary classification (i.e., lateralsteep-type OPLL versus lateral-gentle-type OPLL). There was no statistically significant difference in JOARR between the ipsilateral group and the contralateral group in terms of the lateral-gentle-type OPLL and primary lateral-type OPLL. In lateral-steep-type OPLL, the difference was significant. This is consistent with the results reported by Tang et al. (15), who found that the contralateral group had a higher recovery rate. The increased space within the spinal canal appears to open the door in the same manner; however, the increased space is similar in shape to a fan, as it has a large space on the side of the open door and a much smaller space on the other side (see Figure 4). When there is 

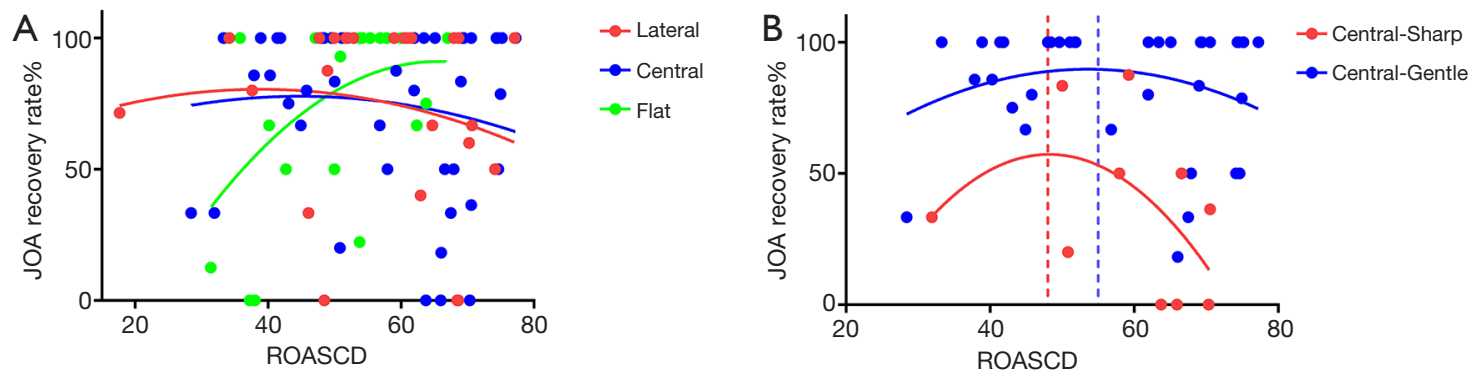

Figure 5 Correlation between the ratio of ossification to axial spinal canal diameter (ROASCD) and recovery rate in different types of OPLL. (A) Trend in primary classification. (B) Trends in central subdivision.

a significant difference in the ossification observed on each side, the spinal cord forms a clear unilateral shape to avoid ossification, and the extrusion pressure on the spinal cord that results tends toward that side. If the door is opened on the healthy side, the space available to the spinal cord after expansion is greater, while on the ossification side, it is much more limited. However, no significant difference was found in lateral-gentle-type OPLL, as the extrusion pressure on the spinal cord was essentially centralized, and the increased space of the central section was similar regardless of which side had the open-door. Thus, we recommend that the contralateral side should be the first choice for an open-door laminoplasty, especially for patients with lateral-steep-type OPLL.

It should be noted that the JOARR and post-operative JOA of flat-type OPLL, which is an independent type of OPLL under our MACTC scheme, did not differ significantly to those of the other primary types, but this type did have a significantly higher ROASCD than that of the other two types. It is well known that the severity of ossification is closely linked to successful prognoses (16). As this was retrospective study, the choice of data could not be controlled. The average ossification of flat-type OPLL was relatively significant; however, the JOARR had a similar value to that of the other two types. Thus, it may be that flat-type OPLL will ultimately obtain a better prognosis than other types; however, more evidence is required to properly corroborate this tentative conjecture.

Additionally, severe OPLL (which accounts for over $60 \%$ of cases) is usually regarded as a strong contraindication for the use of laminoplasty (17). A significant reduction in the recovery rate was also observed when ossification was approximately $50 \%$. Notably, this turning point occurred earlier in central-type OPLL than other types of OPPL (see Figure 5). It is a widely believed that combined posterior-anterior surgery should be performed in severe cases; otherwise, the prognosis will be poor (18). However, the cost of combined surgery places a large financial burden on economically disadvantaged patients. Spinal decompression using laminoplasty, followed by rehabilitation training, represents a less costly alternative. If the symptoms are not sufficiently relieved by this procedure, the patient should then be admitted for additional anterior surgery. The HYR rates of severe OPLL patients was significantly lower than that of mild patients; however, there was no statistically significant difference in OYR rates between the two groups. During the follow-up appointments, there were five cases of patients with persistent, unrelieved, or reappearing symptoms, four cases of severe OPLL, and one case of mild OPLL. Of these patients, three were readmitted for treatment, one due to independent ossification, and two due to high-level thoracic spinal stenosis. The mean annual growth rate of OPLL is $8.00 \% \pm 13.06 \%$ (19), and the average readmission time for ossification growth after the first laminoplasty is 10 years (20). Thus, severe OPLL should not be an absolute contraindication for laminoplasty. We suggest that posterior laminoplasty should be performed first for economically disadvantaged patients, and that 1 year be set as the standard time at which the need for subsequent anterior decompression should be evaluated.

Using the clinical data presented in the current study, we evaluated the accuracy and utility of two established OPLL classifications and their concomitant success in prognosis. Matsuyama (9) proposed that OPLL should be classified according to the shape of the spinal cord compression visualized in T2 axial MRI in order to generate a disease prognosis. In our study, prognosis using the teardrop classification was the most successful, followed 
by boomerang type, while prognosis of triangular type OPLL was the least successful. The results of the current study are not consistent with those of the classification scheme proposed by Matsuyama. The JOARR of the three types was similar, but no statistically significant difference between the three types was found. Further, we identified an OPLL type that had not been described previously. In patients with severe ossification, the spinal cord is linear in type, and compression is more severe than that seen in triangular OPLL. As cases of such severe ossification are rare in developed areas, only seven cases could be examined; however, these cases were reported to supplement the above classification.

Heterogeneous classification methods have previously used several classification systems to describe the morphological features of OPLL, and these systems have described different types of OPLL, such as hill, plateau, mushroom, irregular, square, and triangular $(21,22)$. In the current study, no statistically significant difference was found in the accuracy of prognosis between groups based on the classification scheme proposed by Ono (8). Advanced three-dimensional (3D) CT classification of OPLL provides a more detailed and nuanced picture of the ossified ligament (23). However, it remains similar to the onedimensional (1D) sagittal classification that can be obtained using CT, which does not provide helpful guidance for treatment, is considerably more expensive at the point of use, and is thus not widely accepted among patients. Previous results show that use of 1D, two-dimensional (2D) and 3D methods had little effects on the JOA for OPLL (24).

The OPLL classification proposed in this paper is a synthesis of existing classification schemes. It not only describes the morphology of ossification, but also allows for accurate prognosis and provides guidance in relation to the choice of operative procedures, thus addressing the apparent shortcomings of existing classification schemes thereby. However, our study had a number of limitations. First, bias could not be completely eliminated due to the retrospective nature of our analysis. Second, the sample size was limited due to the low incidence of OPLL and certain forms of the disease. The statistical method employed was suitable for the study; however, the statistical discrepancy was not reliable in some cases due to the small sample size. A prospective cohort study with a larger sample size needs to be conducted to further evaluate and validate the reliability of MACTC. Further, as only cases of multilevel OPLL that met the criteria for use of the posterior approach were included in the present study, there may be some appreciable deviation from prognoses generated by established classification schemes. Thus, this paper only purports to assess the accuracy of existing classifications in predicting the effect of posterior unilateral open-door laminoplasty on OPLL.

\section{Conclusions}

MACTC can assist surgeons in choosing the most appropriate operative procedure and performing disease prognosis. Operations on central-sharp-type OPLL should be performed using a combination of the posterior and anterior approaches; otherwise, prognosis will be poor. The contralateral side should be the first choice for door opening during a laminoplasty, especially for lateral-steeptype OPLL. Different opening sides for lateral-gentle-type ossification have similar prognoses. Severe OPLL may not be an absolute contraindication for the posterior approach. Posterior laminoplasty surgery should be undertaken first for economically disadvantaged patients, who should then be examined 1 year later so that any resulting effects can be observed. If the initial surgery is deemed unsatisfactory, an anterior revision operation may then be required.

\section{Acknowledgments}

We would like to thank Mrs. Sirui Xu for her assistance with the follow-up of patients.

Funding: This research was supported by the Heilongjiang Renxin Foundation.

\section{Footnote}

Conflicts of Interest: All authors have completed the ICMJE uniform disclosure form (available at http://dx.doi. org/10.21037/qims-20-862). Dr. ZY has reported the grants he received from the Heilongjiang Renxin Foundation during the conduct of the study. The other authors have no conflicts of interest to declare.

Ethical statement: The study was conducted in accordance with the Declaration of Helsinki (as revised in 2013). The study was approved by the ethics committee of the First Affiliated Hospital of Harbin Medical University (no. 202063), and individual consent for this retrospective analysis was waived.

Open Access Statement: This is an Open Access article 
distributed in accordance with the Creative Commons Attribution-NonCommercial-NoDerivs 4.0 International License (CC BY-NC-ND 4.0), which permits the noncommercial replication and distribution of the article with the strict proviso that no changes or edits are made and the original work is properly cited (including links to both the formal publication through the relevant DOI and the license). See: https://creativecommons.org/licenses/by-nc-nd/4.0/.

\section{References}

1. Nam DC, Lee HJ, Lee CJ, Hwang SC. Molecular Pathophysiology of Ossification of the Posterior Longitudinal Ligament (OPLL). Biomol Ther (Seoul) 2019;27:342-8.

2. Boody BS, Lendner M, Vaccaro AR. Ossification of the posterior longitudinal ligament in the cervical spine: a review. Int Orthop 2019;43:797-805.

3. Yoshii T, Morishita S, Inose H, Yuasa M, Hirai T, Okawa A, Fushimi K, Fujiwara T. Comparison of Perioperative Complications in Anterior Decompression With Fusion and Posterior Decompression With Fusion for Cervical Ossification of the Posterior Longitudinal Ligament: Propensity Score Matching Analysis Using a NationWide Inpatient Database. Spine (Phila Pa 1976) 2020;45:E1006-12.

4. Xu P, Zhuang JS, Huang YS, Tu C, Chen JT, Zhong ZM. Surgical outcomes of cervical myelopathy due to ossification of posterior longitudinal ligament: Anterior decompression and fusion versus posterior laminoplasty. J Orthop Surg (Hong Kong) 2019;27:2309499019837907.

5. Qin R, Chen X, Zhou P, Li M, Hao J, Zhang F. Anterior cervical corpectomy and fusion versus posterior laminoplasty for the treatment of oppressive myelopathy owing to cervical ossification of posterior longitudinal ligament: a meta-analysis. Eur Spine J 2018;27:1375-87.

6. Li S, Zhang P, Gao X, Miao D, Gao Y, Shen Y. Potential risk factors for poor outcome after anterior surgery for patients with cervical ossification of the posterior longitudinal ligament. Ther Clin Risk Manag 2018;14:341-7.

7. Kawaguchi $Y$, Matsumoto M, Iwasaki M, Izumi T, Okawa A, Matsunaga S, Chiba K, Tsuji T, Yamazaki M, Fujimori T, Yoshii T, Toyama Y. New classification system for ossification of the posterior longitudinal ligament using CT images. J Orthop Sci 2014;19:530-6.

8. Ono S, Uwada O, Tamura S, Watanabe K. CT findings of ossification of the posterior longitudinal ligament
(OPLL), with special emphasis on the relationship to clinical symptoms. Nihon Igaku Hoshasen Gakkai Zasshi 1989;49:1517-24.

9. Matsuyama Y, Kawakami N, Yanase M, Yoshihara H, Ishiguro N, Kameyama T, Hashizume Y. Cervical myelopathy due to OPLL: clinical evaluation by MRI and intraoperative spinal sonography. J Spinal Disord Tech 2004;17:401-4.

10. Hirabayashi K, Watanabe K, Wakano K, Suzuki N, Satomi K, Ishii Y. Expansive open-door laminoplasty for cervical spinal stenotic myelopathy. Spine (Phila Pa 1976) 1983;8:693-9.

11. Matsunaga S, Nakamura K, Seichi A, Yokoyama T, Toh S, Ichimura S, Satomi K, Endo K, Yamamoto K, Kato Y, Ito T, Tokuhashi Y, Uchida K, Baba H, Kawahara N, Tomita $\mathrm{K}$, Matsuyama Y, Ishiguro N, Iwasaki M, Yoshikawa H, Yonenobu K, Kawakami M, Yoshida M, Inoue S, Tani T, Kaneko K, Taguchi T, Imakiire T, Komiya S. Radiographic predictors for the development of myelopathy in patients with ossification of the posterior longitudinal ligament: a multicenter cohort study. Spine (Phila Pa 1976) 2008;33:2648-50.

12. Khuyagbaatar B, Kim K, Purevsuren T, Lee SH, Kim YH. Biomechanical Effects on Cervical Spinal Cord and Nerve Root Following Laminoplasty for Ossification of the Posterior Longitudinal Ligament in the Cervical Spine: A Comparison Between Open-Door and Double-Door Laminoplasty Using Finite Element Analysis. J Biomech Eng 2018;140:10.

13. Imagama $S$, Ando K, Ito Z, Kobayashi K, Hida T, Ito $\mathrm{K}$, Tsushima M, Ishikawa Y, Matsumoto A, Morozumi M, Tanaka S, Machino M, Ota K, Nakashima H, Wakao N, Nishida Y, Matsuyama Y, Ishiguro N. Risk Factors for Ineffectiveness of Posterior Decompression and Dekyphotic Corrective Fusion with Instrumentation for Beak-Type Thoracic Ossification of the Posterior Longitudinal Ligament: A Single Institute Study. Neurosurgery 2017;80:800-8.

14. Matsuyama Y, Yoshihara H, Tsuji T, Sakai Y, Yukawa Y, Nakamura H, Ito K, Ishiguro N. Surgical outcome of ossification of the posterior longitudinal ligament (OPLL) of the thoracic spine: implication of the type of ossification and surgical options. J Spinal Disord Tech 2005;18:492-7; discussion 498.

15. Tang Y, Zhou S, Wang Z, Huang W, Jia L, Chen $X$. Choice of the Open Side in Unilateral OpenDoor Laminoplasty for Cervical Ossification of the Posterior Longitudinal Ligament. Spine (Phila Pa 1976) 
2020;45:741-6.

16. Jung JM, Chung CK, Kim CH, Choi Y. Risk Factors and Prognosis for Acute Progression of Myelopathic Symptoms in Patients Ossification of the Posterior Longitudinal Ligament After Minor Trauma. Spine (Phila Pa 1976) 2018;43:E171-6.

17. Zhang J, Liang Q, Qin D, Song J, An Q, Wang X, Zhao $Z$. The anterior versus posterior approach for the treatment of ossification of the posterior longitudinal ligament in the cervical spine: A systematic review and meta-analysis. J Spinal Cord Med 2019. doi: 10.1080/10790268.2019.1692179. [Epub ahead of print].

18. An HS, Al-Shihabi L, Kurd M. Surgical treatment for ossification of the posterior longitudinal ligament in the cervical spine. J Am Acad Orthop Surg 2014;22:420-9.

19. Lee JJ, Shin DA, Yi S, Kim KN, Yoon DH, Shin HC, Ha Y. Effect of posterior instrumented fusion on threedimensional volumetric growth of cervical ossification of the posterior longitudinal ligament: a multiple regression analysis. Spine J 2018;18:1779-86.

20. Nakashima H, Kanemura T, Satake K, Ito K, Ouchida J, Morita D, Ando K, Kobayashi K, Ishiguro N, Imagama S. Reoperation for Late Neurological Deterioration After Laminoplasty in Individuals With Degenerative Cervical
Myelopathy: Comparison of Cases of Cervical Spondylosis and Ossification of the Posterior Longitudinal Ligament. Spine (Phila Pa 1976) 2020;45:E909-16.

21. Ito K, Yukawa Y, Ito K, Machino M, Kanbara S, Nakashima H, Hida T, Ishiguro N, Imagama S, Kato F. Dynamic changes in the spinal cord cross-sectional area in patients with myelopathy due to cervical ossification of posterior longitudinal ligament. Spine J 2015;15:461-6.

22. Iwasaki M, Okuda S, Miyauchi A, Sakaura H, Mukai Y, Yonenobu K, Yoshikawa H. Surgical strategy for cervical myelopathy due to ossification of the posterior longitudinal ligament: Part 1: Clinical results and limitations of laminoplasty. Spine (Phila Pa 1976) 2007;32:647-53.

23. Wang Z, Sun Y, Tang Y, Yuan B, Zhou S, Chen X, Jia L. Assessment of Myelopathy in Cervical Ossification of the Posterior Longitudinal Ligament by Magnetic Resonance Imaging-Assisted 3-Dimensional Measurement. World Neurosurg 2018;115:e172-7.

24. An SB, Lee JJ, Kim TW, Lee N, Shin DA, Yi S, Kim KN, Yoon DH, Ha Y. Evaluating the differences between 1D, $2 \mathrm{D}$, and $3 \mathrm{D}$ occupying ratios in reflecting the JOA score in cervical ossification of the posterior longitudinal ligament. Quant Imaging Med Surg 2019;9:952-9.
Cite this article as: Shao T, Gu J, Zhu Y, Tang W, Li Q, Lu J, Hu Y, Yu Z, Shen H. Modified axial computed tomography classification of cervical ossification of the posterior longitudinal ligament: selecting the optimal operating procedure and enhancing the accuracy of prognosis. Quant Imaging Med Surg 2021;11(5):1888-1898. doi: 10.21037/qims-20-862 